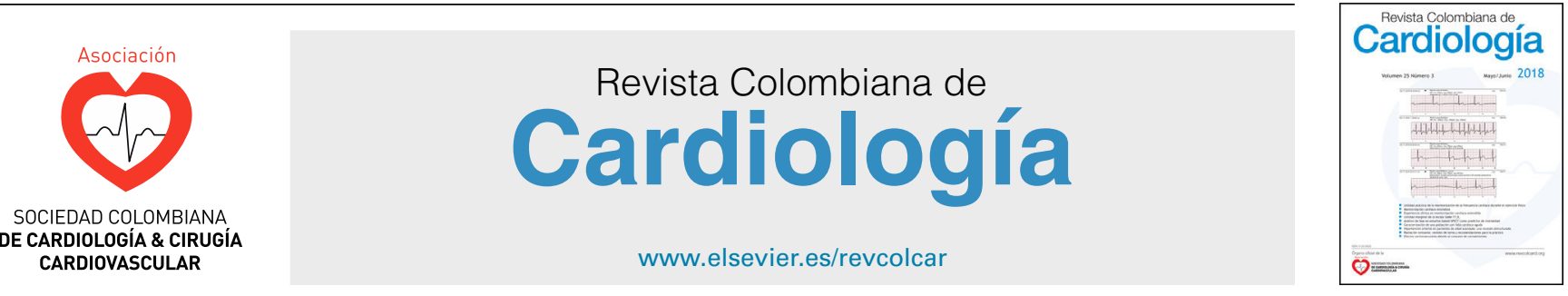

CARDIOLOGÍA DEL ADULTO - ARTÍCULO ORIGINAL

\title{
Predictores de hospitalización prolongada en pacientes con insuficiencia cardiaca aguda
}

\author{
Juan Sebastián Parada Zuluaga a ${ }^{a}$ Kelly Marisancén Carrasquilla ${ }^{a}$, \\ Aura María Vélez Granda ${ }^{a}$, C.I. Saldarriaga Giraldo ${ }^{\text {,** }}$, \\ Álvaro Mauricio Quintero Ossa ${ }^{\mathrm{b}}$, Eliana Mabel Cañas Arenas ${ }^{\mathrm{a}}$ \\ y Adriana María Agudelo ${ }^{c}$
}

\author{
a Facultad de Medicina, Universidad Pontificia Bolivariana, Medellín, Colombia \\ b Departamento de Cardiología, Clínica Cardiovascular Santa María (CardioVID), Universidad Pontificia Bolivariana y Universidad \\ de Antioquia, Medellín, Colombia \\ c Departamento de Enfermería, Clínica Cardiovascular Santa María (CardioVID), Medellín, Colombia
}

Recibido el 4 de julio de 2017; aceptado el 22 de junio de 2018

Disponible en Internet el 15 de diciembre de 2018

\section{PALABRAS CLAVE \\ Falla cardiaca; \\ Gestión sanitaria; \\ Estado de salud; \\ Estancia hospitalaria}

\begin{abstract}
Resumen
Introducción: la insuficiencia cardiaca aguda es la principal causa de hospitalización en adultos mayores de 65 años. La duración de las hospitalizaciones es un determinante del incremento en los costos por la atención hospitalaria.

Objetivo: describir el perfil clínico de los pacientes hospitalizados por insuficiencia cardiaca aguda en un centro de referencia cardiovascular e identificar la duración de la estancia hospitalaria y los predictores de una hospitalización prolongada.

Métodos: estudio observacional analítico, cohorte, prospectivo.

Resultados: durante siete meses se incluyeron 251 pacientes con diagnóstico de insuficiencia cardiaca aguda. La mediana de edad fue de 71 años, fracción de eyección del ventrículo izquierdo de 25\%, clasificación Nohria-Stevenson: húmedo-caliente 78,9\%; húmedo-frío 15,1\%; seco-caliente 2,8\% y seco-frío 2,8\%. El 15,9\% de los pacientes requirieron inotrópicos y 1,2\% vasopresores. Las complicaciones más frecuentes fueron el desarrollo de enfermedad renal aguda $33,1 \%$ y fibrilación auricular de novo 5,2\%. La mediana de estancia hospitalaria fue de 5 días y el $65,7 \%$ presentó estancia prolongada ( $\geq 7$ días). El análisis bivariado mostró predictores de estancia prolongada como requerimiento inotrópico (RR 2,41; IC 95\% 1,77-3,27 p 0,000), clasificación Nohria-Stevenson seco-frío y húmedo-frío (RR 1,86; I 95\%. 1,33-2,61 p 0,001), clasificación NYHA III-IV (RR 1,85; IC 95\% 1,06-3,24 p 0,017), enfermedad renal aguda (RR 1,82; IC $95 \% 1,31-2,55$ p 0,000) y diabetes mellitus (RR 1,47; IC 95\% 1,05-2,06 p 0,026).
\end{abstract}

\footnotetext{
* Autor para correspondencia.

Correo electrónico: clarais@une.net.co (C.I. Saldarriaga Giraldo).
} 
Conclusión: en una población con predominio de función cardíaca reducida y múltiples comorbilidades, la mediana de hospitalización por insuficiencia cardiaca aguda fue de 5 días. Se identificaron como predictores de estancia prolongada el soporte inotrópico, la clasificación Nohria-Stevenson seco-frío y húmedo-frío, NYHA III-IV, la enfermedad renal aguda y la diabetes mellitus.

(c) 2018 Sociedad Colombiana de Cardiología y Cirugía Cardiovascular. Publicado por Elsevier España, S.L.U. Este es un artículo Open Access bajo la licencia CC BY-NC-ND (http:// creativecommons.org/licenses/by-nc-nd/4.0/).

\section{KEYWORDS}

Heart failure; Health care management; Health status; Hospital stay

\section{Predictors of prolonged hospital stay in patients with acute heart failure}

\begin{abstract}
Introduction: Acute heart failure is the main cause of hospital admission in adults over 65 years-old. The length of the hospital stay is a determining factor in the increase in the costs of hospital care.

Objective: To describe the clinical profile of patients admitted to hospital Cardiovascular Reference Centre due to acute heart failure and to determine the duration of the hospital stay and the predictors of prolonged admission.

Methods: A prospective, analytical, observational, cohort study.

Results: A total of 251 patients with a diagnosis of acute heart failure were admitted during a six-month period. The median age was 75 years, with a mean left ventricle ejection fraction of $25 \%$, and a Nohria-Stevenson classification: wet-hot $78.9 \%$; wet-cold $15.1 \%$; dry-hot $2.8 \%$, and dry-cold $2.8 \%$. Inotropes were required by $15.9 \%$ of patients and vasopressors by $1.2 \%$. The most frequent complications were development of acute kidney disease in $33.1 \%$, and de novo atrial fibrillation in $5.2 \%$. The median hospital stay was 5 days, and $65.7 \%$ had a prolonged stay ( $\geq 7$ days). The bivariate analysis showed prolonged stay predictors such as inotrope requirement (RR 2.41; $95 \% \mathrm{Cl} ; 1.77-3.27, P=.000)$, a Nohria-Stevenson classification of dry-cold and wet-cold (RR 1.86; 95\% Cl; 1.33-2.61, $P=.001$ ), NYHA classification of III-IV (RR 1.85; 95\% Cl; 1.06-3.24, $P=.017$ ), acute kidney disease (RR 1.82; 95\% Cl; 1.31-2.55, $P=.000$ ), and diabetes mellitus (RR 1.47; 95\% Cl; 1.05-2.06, $P=.026)$.

Conclusion: In a population with a predominance of reduced cardiac function and multiple comorbidities, the median hospital stay due to acute heart failure was 5 days. Predictors of a prolonged stay were identified as inotrope support, a Nohria-Stevenson classification of dry-cold and wet-cold, NYHA III-IV, acute kidney disease, and diabetes mellitus.

(c) 2018 Sociedad Colombiana de Cardiología y Cirugía Cardiovascular. Published by Elsevier España, S.L.U. This is an open access article under the CC BY-NC-ND license (http:// creativecommons.org/licenses/by-nc-nd/4.0/).
\end{abstract}

\section{Introducción}

La insuficiencia cardiaca es un síndrome clínico causado por disfunción cardiaca estructural o funcional que puede ser secundario a trastornos adquiridos o hereditarios ${ }^{1}$, clínicamente evidenciado por signos y síntomas de baja perfusión y congestión ${ }^{2}$. Existen medicamentos y estrategias útiles para este síndrome, como diuréticos, inotrópicos, vasopresores, vasodilatadores, betabloqueadores, oxigenoterapia, ventilación, entre otros ${ }^{3}$. Las enfermedades cardiovasculares son las responsables del 30\% de las muertes anuales en el mundo ${ }^{4}$, destacándose entre ellas la tasa de mortalidad por insuficiencia cardíaca aguda tanto durante como después de una hospitalización ${ }^{5}$. En Latinoamérica, la enfermedad cardiovascular es la principal causa de mortalidad, y se estima que en las próximas tres décadas las muertes debido a ésta incrementarán en un $200 \%{ }^{6}$. Igualmente en Colombia, este grupo de enfermedades representa la primera causa de mortalidad.

La insuficiencia cardíaca se considera un problema de salud actual, ya que presenta alta morbilidad y mortalidad; la prevalencia mundial es cercana al $2 \%$ en la población adulta y alcanza hasta un $10 \%$ en personas mayores de 70 años. En Europa es del 0,4 al $2 \%$, cifras equiparables a las de Estados Unidos, 2 al 6\%, y a las de Colombia, 2,3\%, de acuerdo con el reporte del Ministerio de Salud de $2012^{6-8}$.

Existen factores relacionados con el paciente y con la enfermedad, así como factores iatrogénicos que pueden agravar los signos y síntomas de la insuficiencia cardíaca, por lo cual suelen requerir ingreso hospitalario ${ }^{1}$. La hospitalización por descompensación de una falla cardíaca es frecuente en pacientes con fracción de eyección del ventrículo izquierdo tanto disminuida como conservada; se estima que la mortalidad intrahospitalaria de estos pacientes es del 4 al $7 \%$. 
En diferentes estudios se ha reportado que el promedio de la duración de la estancia hospitalaria en estos pacientes varía de acuerdo con múltiples factores, ya sean inherentes al paciente o externos a él. La importancia de conocer estos factores se encuentra en la asociación que existe entre la estancia hospitalaria prolongada, diferentes comorbilidades y la mortalidad ${ }^{6}$.

Por tanto, en este estudio se hará una descripción del perfíl clínico de pacientes con insuficiencia cardiaca aguda atendidos en un centro de referencia cardiovascular de Colombia, con miras a identificar la duración promedio de la estancia hospitalaria y los factores asociados a la prolongación de la misma.

\section{Materiales y métodos}

Estudio observacional analítico, de cohorte, prospectivo, en el que se incluyeron adultos con insuficiencia cardiaca aguda que ingresaron a una clínica privada de la ciudad de Medellín en el periodo abril a octubre de 2016, que cumplían criterios de elegibilidad: ser mayores de 18 años, con diagnóstico de insuficiencia cardiaca aguda con fracción de eyección conservada o disminuida, clasificados de acuerdo con la valoración clínica rápida de los síndromes de insuficiencia cardiaca aguda Nohria-Stevenson ${ }^{10}$. Se excluyeron aquellos que requerían cirugía cardiovascular o de cualquier índole y procedimientos hemodinámicos o electrofisiológicos durante su estancia hospitalaria. Las variables evaluadas fueron: número de días de hospitalización (variable dependiente), estancia hospitalaria prolongada (número de días de hospitalización mayor o igual a 7), datos sociodemográficos, reingreso en los últimos seis meses, valoración clínica rápida de los síndromes de insuficiencia cardiaca aguda Nohria-Stevenson, datos ecocardiográficos, uso de inotrópicos, uso de vasopresores, comorbilidades, mortalidad a 30 días y clasificación funcional de acuerdo con la New York Health Association -NYHA-, sistema más utilizado para evaluar la actividad física de los pacientes con enfermedades cardiacas $^{11}$. Se determinó estancia hospitalaria prolongada como una estancia por insuficiencia cardiaca aguda mayor o igual a 7 días.

No se calculó un tamaño muestral; el muestreo fue por conveniencia y se incluyeron todos los pacientes adultos con insuficiencia cardiaca aguda que ingresaron a la clínica Cardiovascular Santa María (CardioVID) en el periodo de estudio. La selección de los pacientes estuvo a cargo de los investigadores, quienes recolectaron la información, y a partir de una fuente secundaria (historias clínicas), extrajeron los datos necesarios. Para esto se hizo uso de un instrumento de recolección con las variables descritas. Adicionalmente, se evaluó el desenlace de mortalidad a los 30 días a través de los datos recolectados en el programa de falla cardiaca de la institución, liderado por una enfermera jefe miembro de la investigación. La descripción de las variables cualitativas se hizo mediante el uso de frecuencias absolutas y relativas, mientras que las variables cuantitativas, como la edad y la estancia hospitalaria, se describieron usando mediana y rango intercuartílico dada la heterogeneidad de la población. Se hizo un análisis bivariado en el que se evaluó la duración en días de la estancia hospitalaria (variable dependiente) con las características sociodemográficas, comorbilidades y demás variables de importancia clínica descritas previamente (variables independientes). Se calcularon los riesgos relativos con sus respectivos intervalos de confianza del $95 \%$ y se consideraron estadísticamente significativos los valores de $\mathrm{p}$ menores a 0,05. La mortalidad se describió en proporción, buscando relación entre la estancia hospitalaria prolongada y el desenlace de mortalidad a 30 días por medio de la prueba de chi-cuadrado.

\section{Resultados}

En un periodo de siete meses, se identificaron 251 pacientes con insuficiencia cardiaca aguda como diagnóstico de ingreso. La mediana de edad fue de 71 (60-80) años, el 55\% de la población eran hombres, el $75 \%$ tenía obesidad (IMC $24 ; 21-27)$ y el $73,7 \%$ pertenecía al régimen contributivo.

La valoración clínica rápida de los síndromes de insuficiencia cardiaca aguda Nohria-Stevenson fue: seco-caliente en un 2,8\%; seco-frío en un 2,8\%; húmedo-frío $15,1 \%$, húmedo-caliente en un $78,9 \%$ (tabla 1 ); este último fue el más frecuente y se asoció con la menor necesidad de soporte inotrópico y vasopresor. Durante la hospitalización, se usaron inotrópicos en un $15,9 \%$ de los pacientes; el más utilizado fue el milrinone en un $49,2 \%$ de los casos que lo requirieron. Se requirió soporte vasopresor en el 1,2\% de los pacientes.

La clase funcional de los pacientes de acuerdo con la clasificación de la New York Heart Association (NYHA), estuvo distribuida de la siguiente manera: clase funcional III $39 \%$, clase funcional IV $35,9 \%$, clase funcional II en un $17,5 \%$, y clase funcional I en $4 \%$.

La mediana de fracción de eyección del ventrículo izquierdo fue $25 \%(20-40,5)$. Su clasificación fue reducida $(\leq 39 \%)$ en un $70,9 \%$, intermedia $(40-49 \%)$ en un $8 \%$ y preservada $(\geq 50 \%$ ) en un $19,1 \%$.

La comorbilidad más frecuente en la población de estudio fue la hipertensión arterial sistémica, seguida en orden descendente por enfermedad renal crónica, fibrilación auricular crónica, enfermedad coronaria, diabetes mellitus, enfermedad pulmonar obstructiva crónica (EPOC) y fibrilación auricular de novo (tabla 2).

Las complicaciones más frecuentes durante la hospitalización fueron el desarrollo de enfermedad renal aguda en el $33,1 \%$ de la población y fibrilación auricular de novo en el $5,2 \%$.

La mediana de duración de la hospitalización por insuficiencia cardiaca aguda fue de 5 días (3-8), y el $65,7 \%$ de la población tuvo una estancia hospitalaria prolongada, es decir, mayor o igual a 7 días. Se determinó que la estancia hospitalaria se consideraría prolongada a partir de siete días, dado que en una publicación colombiana de 2016, la duración promedio de la hospitalización en pacientes con diagnóstico de insuficiencia cardiaca aguda osciló entre 3 y 6 días $^{12}$, y en un centro de referencia cardiovascular en Murcia (España), la mediana de estancia hospitalaria fue de 7 días $^{13}$. No existe una definición única y homogénea para la estancia hospitalaria apropiada, por el contrario, hay gran variabilidad ${ }^{14}$, según se evidencia en la tabla 3. 
Tabla 1 Caracterización sociodemográfica de los pacientes incluidos en el estudio $(n=251)$

\begin{tabular}{|c|c|}
\hline \multicolumn{2}{|l|}{ Cuantitativas, mediana (RIC) } \\
\hline Edad (años) & $71(60-80)$ \\
\hline Peso (kilogramos) & $65(55-73)$ \\
\hline Talla (centímetros) & $162(155-170)$ \\
\hline IMC $^{* \star}\left(\right.$ kilogramos/metro $\left.{ }^{2}\right)$ & $24(21,4-27,5)$ \\
\hline Estancia hospitalaria (días) & $5(3-8)$ \\
\hline \multicolumn{2}{|l|}{ Hallazgos ecocardiográficos } \\
\hline FEVI ๆ $(\%)$ & $25(20-40)$ \\
\hline \multicolumn{2}{|l|}{ Diámetros ventriculares (cm) } \\
\hline Ventrículo izquierdo en sístole & $5(3,8-5,7)$ \\
\hline Ventrículo izquierdo en diástole & $5,8(4,8-6,6)$ \\
\hline Ventrículo derecho & $3,7(2,1-4,6)$ \\
\hline \multicolumn{2}{|l|}{ Tamaño de aurícula izquierda } \\
\hline Bidimensional $\left(\mathrm{cm}^{2}\right)$ & $27(23-33)$ \\
\hline Volumen $\left(\mathrm{ml} / \mathrm{m}^{2}\right)$ & $58(43-69,5)$ \\
\hline \multicolumn{2}{|l|}{ Creatinina $(\mathrm{mg} / \mathrm{dl})$} \\
\hline Basal & $1,1(0,9-1,39)$ \\
\hline Ingreso & $1,18(0,95-1,55)$ \\
\hline Control & $1,2(0,97-1,56)$ \\
\hline \multicolumn{2}{|l|}{ Cualitativas, n (\%) } \\
\hline Sexo (masculino) & $138(55)$ \\
\hline Tipo de régimen de salud (contributivo) & $185(73,7)$ \\
\hline \multicolumn{2}{|l|}{ Perfil clínico de la insuficiencia cardiaca aguda Nohria-Stevenson } \\
\hline Seco-Caliente & $7(2,8)$ \\
\hline Seco-Frío & $7(2,8)$ \\
\hline Húmedo-Caliente & $198(78,9)$ \\
\hline Húmedo-Frío & $38(15,1)$ \\
\hline \multicolumn{2}{|l|}{ Clasificación NYHA ${ }^{\dagger}$} \\
\hline I & $10(4,0)$ \\
\hline II & $44(17,5)$ \\
\hline III & $98(39,0)$ \\
\hline IV & $90(35,9)$ \\
\hline Antecedente de más de una hospitalización por insuficiencia cardiaca aguda en los últimos seis meses & $138(55)$ \\
\hline Estancia hospitalaria prolongada ( $\geq 7$ días) & $165(65,7)$ \\
\hline Mortalidad a 30 días del egreso hospitalario & $23(9,2)$ \\
\hline \multicolumn{2}{|l|}{ Clasificación de acuerdo a la FEVI (porcentaje) } \\
\hline Preservada & $48(19,1)$ \\
\hline Intermedia & $20(8)$ \\
\hline Reducida & $178(70,9)$ \\
\hline Soporte inotrópico-vasopresor & $43(17,2)$ \\
\hline Dobutamina & $6(2,4)$ \\
\hline Milrinone & $19(7,6)$ \\
\hline Levosimendan & $2(0,8)$ \\
\hline Terapia combinada & $13(5,2)$ \\
\hline Noradrenalina & $3(1,2)$ \\
\hline $\begin{array}{l}\text { * RIC: rango intercuartílico } \\
\text { ** Índice de masa corporal } \\
\text { I Fracción de eyección del ventrículo izquierdo } \\
\dagger \text { New York Health Association }\end{array}$ & \\
\hline
\end{tabular}

El $55 \%$ del total de los pacientes incluidos presentó una o más hospitalizaciones previas. Finalmente, la mortalidad a los 30 días del egreso hospitalario fue del $9,2 \%$.

El análisis bivariado (tabla 4) identificó como predictores de estancia prolongada el requerimiento inotrópico (RR 2,41; IC 95\% 1,77-3,27 p 0,000), la valoración clínica rápida de los síndromes de insuficiencia cardiaca aguda Nohria-Stevenson seco-frío y húmedo- frío (RR 1,86; IC 95\% 1,33-2,61 p 0,001), la clasificación NYHA III-IV (RR 1,85; IC 95\% 1,06-3,24 p 0,017), el desarrollo de enfermedad renal aguda (RR 1,82; IC 95\% 1,31-2,55 p 0,000) y el antecedente personal de diabetes mellitus (RR 1,47; IC 95\% 1,05-2,06 p $0,026)$. 
Tabla 2 Frecuencia de comorbilidades $(\mathrm{n}=251)$

\begin{tabular}{ll}
\hline Comorbilidades & $\mathrm{n}(\%)$ \\
\hline Hipertensión arterial sistémica & $199(79,3)$ \\
Enfermedad renal crónica & $161(64,1)$ \\
Fibrilación auricular crónica & $113(45)$ \\
Enfermedad coronaria & $104(41,4)$ \\
Diabetes mellitus & $85(33,9)$ \\
Enfermedad renal aguda & $83(33,1)$ \\
EPOC & $69(27,5)$ \\
Fibrilación auricular de novo & $13(5,2)$ \\
\hline
\end{tabular}

Enfermedad Pulmonar Obstructiva Crónica

\section{Discusión}

Los resultados de este estudio muestran que en una población de pacientes bajo diagnóstico de insuficiencia cardiaca aguda con función cardíaca predominantemente reducida atendidos en un centro de referencia cardiovascular en Colombia, la mediana de estancia hospitalaria fue de 5 días. Así mismo, fueron predictores de estancia hospitalaria prolongada el antecedente de uso de inotrópicos durante la hospitalización, el perfil clínico seco-frío y húmedo-frío de la insuficiencia cardiaca aguda, la clasificación NYHA III-IV, el desarrollo de enfermedad renal aguda durante la estancia hospitalaria y el antecedente personal de diabetes mellitus.
El requerimiento de soporte inotrópico como factor predictor de estancia hospitalaria prolongada se ha descrito en estudios previos, e incluso se ha considerado como un factor que aumenta la mortalidad intrahospitalaria. Adicionalmente, en un análisis que incluyó más de 800 pacientes, se evidenció que comorbilidades como la hipertensión arterial sistémica y la enfermedad renal crónica, se asociaban con mayor probabilidad de recibir tratamiento inotrópico, hallazgo que concuerda con el de este estudio, dado que ambas comorbilidades fueron las más frecuentes en la población incluida ${ }^{15}$. El perfil clínico de la insuficiencia cardiaca aguda seco-frío y húmedo-frío se asocia con la necesidad de soporte inotrópico por el compromiso del estado hemodinámico, caracterizándose por ser una población con mayor morbilidad.

Así mismo, la insuficiencia cardiaca aguda con disfunción sistólica y bajo gasto, representa un estado de hipoperfusión renal que puede generar un deterioro agudo de la función renal, lo cual evidencia una relación entre estos tres factores, que fueron significativos para la prolongación de la estancia hospitalaria.

La enfermedad renal aguda es el deterioro repentino de la función renal, que conlleva aumento del nitrógeno ureico y la creatinina, y en ocasiones se asocia con la reducción del volumen de la orina ${ }^{16}$. El deterioro de la función renal se ha identificado como un factor de riesgo para enfermedad cardiovascular y mortalidad en pacientes con insuficiencia cardiaca $^{17}$ y adicionalmente se ha considerado que

Tabla 3 Duración de la estancia hospitalaria en diferentes artículos publicados

\begin{tabular}{lll}
\hline Autores & País/año & Duración media de la estancia hospitalaria (días) \\
\hline Hermida et $\mathrm{al}^{9}$. & España / 2008 & 12,9 \\
Senior et $\mathrm{al}^{32}$. & Colombia / 2011 & 11,42 \\
Cowie et $\mathrm{al}^{19}$. & Países europeos / 2006 & 9 \\
Saldarriaga et $\mathrm{al}^{12}$. & Colombia / 2016 & 5,9 \\
Alcalá et $\mathrm{al}^{18}$. & México / 2015 & 4,27 \\
Reynolds et $\mathrm{al}^{26}$. & Estados Unidos / 2015 & 3,8 \\
\hline
\end{tabular}

Tabla 4 Análisis bivariado. Estancia hospitalaria prolongada ( $\geq 7$ días) con características sociodemográficas, comorbilidades y demás variables de importancia clínica

\begin{tabular}{lll}
\hline Variable & $\mathrm{RR}, \mathrm{IC} 95 \%$ & $\mathrm{P}$ \\
\hline Requerimiento inotrópico & $2,41(1,77-3,27)$ & 0,000 \\
Valoración clínica rápida de los síndromes de & $1,86(1,33-2,61)$ & 0,001 \\
$\quad$ insuficiencia cardiaca aguda & & \\
$\quad$ Nohria-Stevenson & & 0,017 \\
Clasificación NYHA III y IV & $1,85(1,06-3,24)$ & 0,000 \\
Insuficiencia renal aguda & $1,82(1,31-2,55)$ & 0,026 \\
Diabetes mellitus & $1,47(1,05-2,06)$ & 1,567 \\
Sexo (mujer) & $1,27(0,90-1,80)$ & 0,645 \\
EPOC & $1,09(0,75-1,59)$ & 0,788 \\
Hipertensión arterial sistémica & $1,06(0,68-1,63)$ & 0,910 \\
Insuficiencia renal crónica & $0,98(0,68-1,40)$ & 0,073 \\
Enfermedad coronaria & $0,71(0,49-1,04)$ & 0,100 \\
Fibrilación auricular (crónica y de novo) & $0,7(0,52-1,06)$ \\
\hline$*$ New York Health Association & &
\end{tabular}


aumenta el riesgo de duración prolongada de la estancia hospitalaria ${ }^{18-20}$. Un estudio en pacientes con insuficiencia cardiaca y fracción de eyección reducida, concluyó que aquellos con deterioro de la función renal, entendido en ese estudio como un aumento de la creatinina mayor a $0,3 \mathrm{mg} / \mathrm{dl}$, tuvieron dos días más de estancia hospitalaria frente a quienes no desarrollaron esta condición ${ }^{19}$. Un $20 \%$ de los pacientes con insuficiencia cardiaca aguda incluidos en un estudio realizado entre los años 2008 y 2010, cursó con deterioro de la función renal, lo que sugiere que esta alteración es un factor frecuente en este grupo de pacientes ${ }^{20}$.

Es preciso resaltar que previamente se ha descrito una relación entre la enfermedad renal aguda y el requerimiento de inotrópicos. La enfermedad renal aguda se ha considerado un predictor fuerte de necesidad de manejo con inotrópicos, ya que al incrementar el gasto cardiaco estos medicamentos mejoran la perfusión renal y facilitan la diuresis $^{15}$. En este estudio ambos fueron considerados predictores para estancia hospitalaria prolongada, de modo que surge la inquietud acerca de si ambos factores aisladamente prolongan la estancia, o si por el contrario, la prolongación de la estancia se debe a uno de estos.

La fibrilación auricular afecta millones de personas al igual que la insuficiencia cardiaca y su prevalencia aumenta de forma notable con la edad ${ }^{21,22}$. Sin embargo, no se encontró asociación de riesgo entre estancia hospitalaria prolongada y esta enfermedad, pese a ser reportada como factor de riesgo por Mendes et al. ${ }^{23}$. En México, se realizó un estudio en el cual se exploraron factores asociados a la prolongación de la estancia hospitalaria en pacientes mayores de 65 años, entre los cuales se hallaron: fibrilación auricular, días de ingreso, tabaquismo, clase funcional y estado de dependencia funcional con el índice de Barthel ${ }^{18}$. En otro estudio hecho en España, se determinaron factores que aumentaron el riesgo de prolongación de la estancia hospitalaria, como enfermedad renal crónica, cardiopatía isquémica e hipertensión arterial ${ }^{24}$, condiciones que no fueron significativas como predictores en este estudio. En el caso de la hipertensión arterial, llama la atención que el análisis bivariado no arrojó esta variable como predictor de estancia hospitalaria prolongada, a pesar de ser una comorbilidad frecuente en la población y que se ha descrito como tal en otros estudios. Se plantean hipótesis respecto a las diferencias en las características de la población de los estudios o por el mismo tamaño de la muestra.

La diabetes mellitus es una comorbilidad que se encuentra hasta en un $30 \%$ de los pacientes con diagnóstico de insuficiencia cardiaca. En este estudio dicha variable se comportó como un factor predisponente para la prolongación de la estancia hospitalaria, lo cual concuerda con lo reportado en la literatura ${ }^{14}$.

Es importante mencionar que no todos los factores modifican la estancia hospitalaria, como la variable sexo, ya que se ha reportado que la duración de la hospitalización para hombres y mujeres es similar ${ }^{25}$.

La insuficiencia cardiaca es una enfermedad que genera altos costos, dentro de los cuales se destacan los que se dan a partir de las hospitalizaciones y las readmisiones. Se ha registrado que aproximadamente el $20 \%$ de pacientes con diagnóstico de insuficiencia cardiaca que son dados de alta, reingresan en los siguientes 30 días y un $44 \%$ lo hace dentro de los seis meses siguientes por diferentes causas ${ }^{26}$.
La relevancia de la duración de la estancia hospitalaria radica en su asociación con readmisiones y con la mortalidad. En nuestro estudio se evaluó la mortalidad a 30 días después del egreso hospitalario y se encontró que $9(39,1 \%)$ pacientes con estancia mayor de 7 días, fallecieron por causa cardiovascular en ese período de tiempo. No obstante, no se encontraron diferencias significativas respecto a los que tuvieron una estancia menor de 7 días $(p=0,586)$. Reynolds et al. reportan que la duración de la estancia hospitalaria mayor a 5 días se relaciona con mayor riesgo de readmisión a 30 días y a un año si se compara con una estancia de 3 a 4 días $^{26}$.

Es importante evitar estancias hospitalarias prolongadas en pacientes con diagnóstico de insuficiencia cardiaca aguda que reciben manejo adecuado e interdisciplinario. Las condiciones crónicas se relacionan con mayor número de hospitalizaciones, así que pueden estar en riesgo de incurrir en complicaciones asociadas con la atención hospitalaria. En consecuencia, la prolongación de la estancia hospitalaria acarrea sobrecostos por su manejo y otros eventos que emergen durante la atención, desde medicamentos suplementarios hasta intervenciones quirúrgicas ${ }^{27,28}$.

En términos de mortalidad, estudios han reportado que la insuficiencia cardiaca aguda severa puede ser incluso más maligna que diferentes tipos de cáncer ${ }^{29}$. Los costos que esta enfermedad genera al sistema de salud pueden ser tan altos como los del caso de Norteamérica, donde se acercan a 20 billones de dólares anuales ${ }^{30}$.

La estancia hospitalaria puede ser influenciada por procesos infecciosos concomitantes a la insuficiencia cardiaca aguda. Un estudio realizado en Londres concluyó que la prolongación de la duración de la estancia hospitalaria debido a una infección en el torrente sanguíneo adquirida en el hospital, es de 1,1 días para los microorganismos susceptibles a los antibióticos y de 2,5 días para los resistentes ${ }^{31}$. En nuestro estudio, el abordaje de esta variable fue, que en caso de obtenerse una mediana de estancia hospitalaria no esperada para la institución donde se realizó el estudio (superior a 5.9 días), se procedería a un análisis de subgrupos entre los pacientes con insuficiencia cardiaca aguda con procesos infecciosos concomitantes, y pacientes con insuficiencia cardiaca aguda sin procesos infecciosos concomitantes. El análisis de subgrupos no se llevó a cabo tras hallar la estancia hospitalaria con una mediana esperada para la institución.

Los resultados obtenidos de esta investigación pueden contribuir a la implementación de acciones preventivas para cada grupo de riesgo y planificar los servicios apuntando a la reducción de los días de hospitalización y costos en la atención por esta enfermedad.

\section{Conclusiones}

Los resultados de este estudio de cohorte prospectiva mostraron que en una población de pacientes con insuficiencia cardiaca aguda con función ventricular predominantemente reducida, atendidos en un centro de referencia cardiovascular en Colombia, la mediana de duración de la hospitalización fue de 5 días. En este estudio, se definió una estancia hospitalaria prolongada como una hospitalización mayor o igual a 7 días. 
Se identificaron como predictores de estancia prolongada el requerimiento de tratamiento inotrópico, la clasificación clínica rápida de los síndromes de insuficiencia cardiaca aguda Nohria-Stevenson seco-frío y húmedo-frio, la clasificación NYHA III-IV, la enfermedad renal aguda y el antecedente de diabetes mellitus. Es importante identificar estos factores en pacientes con insuficiencia cardiaca aguda, para implementar así estrategias preventivas y terapéuticas que impacten en desenlaces adversos en esta población.

Se hace un llamado a las instituciones y al personal de la salud, para que fomenten un seguimiento óptimo y un tratamiento oportuno de los pacientes en quienes desde consulta general se identifique falla cardiaca crónica NYHA III-IV y Nohria-Stevenson seco-frío y húmedo-frío, con el objetivo de mejorar su condición clínica y disminuir los costos en la atención de esta población.

\section{Financiación}

Universidad Pontificia Bolivariana.

Cínica Cardiovascular Santa María (CardioVID).

\section{Conflicto de intereses}

\section{Ninguno.}

\section{Reconocimientos y agradecimientos}

A la clínica Cardiovascular Santa María (CardioVID) por su colaboración en el análisis de la información y desarrollo del proyecto.

\section{Bibliografía}

1. Farmakis D, Parissis J, Lekakis J, Filippatos G. Acute heart failure: Epidemiology, risk factors, and prevention. Rev Española Cardiol Engl Ed. 2015;68:245-8.

2. Adams KF, Fonarow GC, Emerman CL, LeJemtel TH, Costanzo MR, Abraham WT, et al. Characteristics and outcomes of patients hospitalized for heart failure in the United States: rationale, design, and preliminary observations from the first 100,000 cases in the Acute Decompensated Heart Failure National Registry (ADHERE). Am Heart J. 2005 febrero de;149:209-16.

3. Mann DL, Chakinala M. Heart Failure: Pathophysiology and Diagnosis. En: Kasper D, Fauci A, Hauser S, Longo D, Jameson JL, Loscalzo J, editores. Harrison's Principles of Internal Medicine. 19. ${ }^{a}$ ed. New York, NY: McGraw-Hill Education; 2015. p. 1500-6.

4. Mendoza A, Alexandra M, Rojas Velasco G, Vieyra Herrera G, Uriarte LAO, et al. Insuficiencia cardíaca aguda e insuficiencia cardíaca descompensada. Arch Cardiol México. 2007;77:27-33.

5. Villero AMU, Castaño KDCM, Criado CAR, Duarte WD. Manejo de la falla cardiaca aguda en urgencias: enfoque terapia farmacológica. Rev MÉDICAS UIS [Internet]. [Acceso 5 Nov 2013]. Disponible en: http: / /revistas.uis.edu.co/ index.php/revistamedicasuis/article/view/3593.

6. Chaves WG, Diaztagle JJ, Sprockel JJ, Hernández JI, Benavidez JM, Henao DC, et al. Factores asociados a mortalidad en pacientes con falla cardiaca descompensada. Acta Méd Colomb. 2014;39:314-20.

7. Jaramillo C, Gómez E, Hernández E, Saldarriaga C, Flórez N, Buitrago R, et al. Consenso Colombiano para el Diagnóstico y Tratamiento de la Insuficiencia Cardiaca Crónica. Primera edición Bogotá: Sociedad Colombiana de Cardiología y Cirugía Cardiovascular; 2014.

8. Hernández-Leiva E. Epidemiology of acute coronary syndrome and heart failure in Latin America. Rev Esp Cardiol. 2011;64 Suppl 2:34-43.

9. Hermida Ameijeiras A, Pazo Núñez M, de la Fuente Cid R, Lado Lado FL, Hernández Fernández L, Rodríguez López I. Epidemiological events related to decompensated heart failure. An Med Interna Madr Spain. 2008;25:256-61.

10. Nohria A, Mielniczuk LM, Warner Stevenson L. Evaluation and monitoring of patients with acute heart failure syndromes. Am J Cardiol. 2005;96:32-40.

11. Asano R, Kajimoto K, Oka T, Sugiura R, Okada H, Kamishima K, et al. Association of New York Heart Association functional class IV symptoms at admission and clinical features with outcomes in patients hospitalized for acute heart failure syndromes. Int J Cardiol. 2017;230:585-91.

12. Saldarriaga Cl, Garcés JJ, Agudelo A, Guarín LF, Mejía J. Impacto clínico de un programa de falla cardiaca. Rev Colomb Cardiol. 2016;23:260-4.

13. Fernández Gassó ML, Hernando-Arizaleta L, Palomar-Rodríguez JA, Soria-Arcos F, Pascual-Figal DA. Trends and characteristics of hospitalization for heart failure in a population setting from 2003 to 2013. Rev Esp Cardiol Engl. 2017;70:720-6.

14. Martin-Sanchez FJ, Carbajosa V, Llorens P, Herrero P, Jacob J, Miró $\mathrm{O}$, et al. Tiempo de estancia prolongado en los pacientes ingresados por insuficiencia cardiaca aguda. Gac Sanit. 2016;30:191-200.

15. Aljundi AHS, Mohammed SFK, Patel A, Singh R, Arabi A, AlBinali $\mathrm{HA}$, et al. Inotropic agents use in patients hospitalized with acute decompensated heart failure: a retrospective analysis from a 22-year registry in a Middle-Eastern Country (1991-2013). BMC Cardiovasc Disord. 2016:16.

16. Waikar SS, Bonventre JV. Acute kidney injury. En: Kasper D, Fauci A, Hauser S, Longo D, Jameson JL, Loscalzo J, editores. Harrison's Principles of Internal Medicine [Internet]. 19. ${ }^{\mathrm{a}}$ ed New York, NY: McGraw-Hill Education; 2015. p. 1799-811. Disponible en: accessmedicine.mhmedical.com/content. aspx?aid $=1120809453$.

17. Kitamura M, Izawa KP, Taniue H, Mimura Y, Ikeda Y, Nagashima $\mathrm{H}$, et al. Activities of daily living at different levels of renal function in elderly hospitalized heart failure patients. Aging Clin Exp Res. 2018;30:45-51.

18. Alcalá-Salgado MÁ, García-Chávez J, Villaseñor-Gómez S, Reynoso-Marenco MT. Factores de riesgo asociados con estancia hospitalaria prolongada en pacientes con insuficiencia cardiaca descompensada. Rev Espec Méd-Quirúrgicas. 2015;20:24-30.

19. Cowie MR, Komajda M, Murray-Thomas T, Underwood J, Ticho B. POSH Investigators. Prevalence and impact of worsening renal function in patients hospitalized with decompensated heart failure: results of the prospective outcomes study in heart failure (POSH). Eur Heart J. 2006;27:1216-22.

20. Abo-Salem E, Sherif K, Dunlap S, Prabhakar S. Potential aetiologies and prognostic implications of worsening renal function in acute decompensated heart failure. Acta Cardiol. 2014;69:657-63.

21. Maisel WH, Stevenson LW. Atrial fibrillation in heart failure: epidemiology, pathophysiology, and rationale for therapy. Am J Cardiol. 2003;91:2-8.

22. Jois-Bilowich P, Michota F, Bartholomew JR, Glauser J, Diercks D, Weber J, et al. Venous thromboembolism prophylaxis in hospitalized heart failure patients. J Card Fail. 2008;14:127-32.

23. Mendes de\#\#SNS, Atié J, Garcia MI, Gripp E de A, Sousa ASde, Feijó LA, et al. Atrial fibrillation in decompensated heart failure: associated factors and in-hospital outcome. Arq Bras Cardiol. 2014;103:315-22.

24. Frigola-Capell E, Comin-Colet J, Davins-Miralles J, GichSaladich I, Wensing M, Verdú-Rotellar JM. Trends and predictors 
of hospitalization, readmissions and length of stay in ambulatory patients with heart failure. Rev Clin Esp. 2013;213:1-7.

25. Galvao M, Kalman J, DeMarco T, Fonarow GC, Galvin C, Ghali JK, et al. Gender differences in in-hospital management and outcomes in patients with decompensated heart failure: analysis from the Acute Decompensated Heart Failure National Registry (ADHERE). J Card Fail. 2006;12:100-7.

26. Reynolds K, Butler MG, Kimes TM, Rosales AG, Chan W, Nichols GA. Relation of Acute Heart Failure Hospital Length of Stay to Subsequent Readmission and All-Cause Mortality. Am J Cardiol. 2015;116:400-5.

27. Cotter G, Davison BA, Milo O, Bourge RC, Cleland JGF, Jondeau G, et al. Predictors and Associations With Outcomes of Length of Hospital Stay in Patients With Acute Heart Failure: Results From VERITAS. J Card Fail. 2016;22:815-22.

28. Nodarse Hernández R. Visión actualizada de las infecciones intrahospitalarias. Rev Cuba Med Mil. 2002;31:201-8.
29. Stewart S, Maclntyre K, Hole DJ, Capewell S, McMurray JJ. More «malignant» than cancer? Five-year survival following a first admission for heart failure. Eur J Heart Fail. 2001;3:315-22.

30. Tavares LR, Victer H, Linhares JM, de Barros CM, Oliveira MV, Pacheco LC, et al. Epidemiology of decompensated heart failure in the city of Niterói: EPICA - Niterói Project. Arq Bras Cardiol. 2004;82:125-8, 121-4.

31. Green N, Johnson AP, Henderson KL, Muller-Pebody B, Thelwall S, Robotham JV, et al. Quantifying the Burden of HospitalAcquired Bloodstream Infection in Children in England by Estimating Excess Length of Hospital Stay and Mortality Using a Multistate Analysis of Linked, Routinely Collected Data. J Pediatr Infect Dis Soc. 2015;4:305-12.

32. Senior JM, Saldarriaga C, Rendón JA. Descripción clínicoepidemiológica de los pacientes con falla cardiaca aguda que consultan al servicio de urgencias. Acta Med Colomb. 2011;36:125-9. 